\title{
TH1B-T5-2
}

\section{Monitoring chemically enhanced transdermal delivery pathways of luminescent quantum dots by multiphoton microscopy}

\author{
Wen Lo ${ }^{1 \#}$, Chien-Hsin Tso ${ }^{1 \#}$, Pei-Yue Huang ${ }^{2}$, Chih-Lung Hsu ${ }^{2}$, Chun-Chia Yeh ${ }^{2}$, \\ Sung-Jan Lin $^{3}$, Shiou-Hwa Jee ${ }^{3,4}$, Chia-Chun Chen ${ }^{2}, \&$ Chen-Yuan Dong ${ }^{1 *}$ \\ 'Department of Physics, National Taiwan University \\ ${ }^{2}$ Department of Chemistry, National Taiwan Normal University \\ ${ }^{3,4}$ Department of Dermatology, National Taiwan University ${ }^{3}$ Hospital and ${ }^{4} \mathrm{Medical}$ College \\ ${ }^{1}$ No.l Roosevelt Road Sec. 4, Taipei, 106, Taiwan \\ ${ }^{2}$ No. 88, Sec. 4, Ting-Chow Rd., Taipei, Taiwan, 116. \\ ${ }^{3}$ No.7, Chung San South Road, Taipei, 100, Taiwan and ${ }^{4}$ No.1, Ren-Ai Rd, 1st Section, Taipei, 100, Taiwan. \\ \# These authors contributed equally to this work \\ * To whom correspondence should be addressed: cydong@phys.ntu.edu.tw
}

\begin{abstract}
Luminescent quantum dots ( $Q D S$ ) are attractive fluorescent probes in bioimaging application In this work, we investigate the feasibility of administering $Q D s$ ( $7 \mathrm{~nm}$ in size) through transdermal delivery pathway using multiphoton fluorescence imaging. Our results show that QDs of this size range can be effectively delivered through intercellular pathways using the chemical enhancer oleic acid. This work has implications for understanding the transport of important biological macromolecules. Our results suggest that QDs can be non-invasively delivered for biological imaging and for probing the transport of nanometer-scale material in tissue. These results also imply that the size of nanoparticles may dominate the nature of transdermal transport mechanism.
\end{abstract}

Introduction

Luminescent nanoparticles become a new class of fluorescent probes for biological applications in recent years[1]. On the one hand, comparing to conventional fluorescent molecules, these semiconducting particles possess several superior optical properties, such as broad excitation spectra, narrow emission spectra and high photochemical stability. The broad excitation spectra provide the feasibility of exciting different colored quantum dots with a single excitation wavelength. In addition, the narrow and tunable emission spectra are two main characters of their emission properties which is size dependent and originate from quantum confinement effect. In addition, since the excitation processes exist in the solid particles, photobleaching can be highly reduced. Furthermore, the size of quantum dots varies from $2 \mathrm{~nm}$ to $20 \mathrm{~nm}$ which overlaps the size range of some important biological macromolecules. The similarity in size and luminescence properties make quantum dots proper probes for monitoring delivery pathways of nanometer-scaled materials.

In this study, we investigate the feasibility of delivering nanoparticles in biological tissues via the non-invasive transdermal delivery pathway. To reduce the resistance of the main barrier of skin, oleic acid is used to facilitate the delivery[2]. It is believed that the kink inherent to structure of oleic acid increase the membrane fluidity when oleic acid molecules are inserted in to the lipid layers. Finally, the delivery pathways were monitored by a two-photon scanning microscope which can image deeply in biological specimens.

\section{Materials and methods}

In this investigation, the delivery pathways of both small organic dye molecule (fluorescein) and nanometer scaled quantum dots are studied. The ex-vivo method is used to investigate the transdermal delivery pathways. A piece of abdominal skin of a mouse was placed between the receiver and donor chambers of a diffusion cell. The upper pool of the diffusion chamber is filled with the diffusion solution described below while the lower tube is filled with PBS.

Fluorescein molecules and hydrophilic quantum dots are mixed and solved in diffusion solution (47.5\% PBS, $47.5 \%$ ethanol and $5 \%$ oleic acid). In the control case, the extra $5 \%$ oleic acid is removed. The concentrations of quantum dots and fluorescein in diffusion solution are $6.25 \mu \mathrm{M}$ and $1.5 \mathrm{mM}$, respectively. Fluorescein molecules $\left(\mathrm{C}_{20} \mathrm{H}_{12} \mathrm{O}_{5}, \mathrm{MW} 332.31\right)$ are used as the representative of smaller organic molecules and the fluorescent emission center is about $514 \mathrm{~nm}$. The quantum dots used in this experiment were synthesized by the chemical colloidal method. A core-shell structure with the core composed of CdSe and the shell composed of $\mathrm{ZnS}$ was formed. Quantum dots that approximate $5 \mathrm{~nm}$ in diameter were selected and the center wavelength of the fluorescence emission is about $600 \mathrm{~nm}$.

The scanning two-photon microscope used in this experiment is composed of an upright microscope and a Ti/Sapphire laser which is a pulsed source and has a distribution of wavelength around $780 \mathrm{~nm}$, a pulse with of around 75 femtosecond and a repetition rate of $80 \mathrm{MHz}$. A water-immersion objective (60X Plan Apo, N.A. 1.2, Nikon $^{\mathrm{TM}}$, Japan ) was used to excite the specimen and collect the fluorescence.

Results

With the arrangement described above, we found that without the oleic acid treatment of the skin, fluorescein 
can still penetrate the skin while the $7 \mathrm{~nm}$ quantum dots did not show significant penetration into the skin. With the addition of $5 \%$ oleic acid, both of them can penetrate deeper (Figure 2). In Figure 1, we show the separate and combined green (fluorescein) and red (quantum dots) fluorescence images of oleic acid treated skin specimen. The fluorescein and quantum dot intensities across a horizontal slice composed of 256 pixels (dashed line) are plotted. The cross-sectional images illustrate several points. First, the skin treated with oleic acid demonstrated a greater degree of quantum dots penetration than the skin without such treatment. Figure 1 also shows a difference in distribution between the fluorescein molecules and the larger quantum dots. Except at the skin surface where the inter- and intracellular regions are not easily identifiable, the cross-sectional analysis shows that the quantum dots are limited to the intercellular regions while the fluorescein molecules are present in both inter- and intracellular domains of the skin. At the depth of $4.2 \mu \mathrm{m}$, the intercellular regions (indicated by arrows) correlate with the peaks in the cross sectional profiles of fluorescein and the quantum dots. To analyze the distribution of fluorescein and the quantum dots in the inter- and intracellular regions, the images were normalized to the peak intensity and the average of the two domains were computed. At the depths of 4.2 $\mu \mathrm{m}$, the normalized inter- and intracellular intensities for fluorescein are $0.64 \pm 0.11$ and $0.29 \pm 0.05$, respectively. The corresponding values for the quantum dots are $0.49 \pm 0.16$ and $0.05 \pm 0.02$. At $8.3 \mu \mathrm{m}$ of depth, the respective values of averaged normalized intensity for fluorescein are $0.65 \pm 0.01$ and $0.32 \pm 0.11$, and they are $0.37 \pm 0.26$ and $0.04 \pm 0.03$ for the quantum dots. Therefore, the smaller fluorescein molecules were able to penetrate into intracellular domains with about half the intensity compared to the intercellular region. On the other hand, our results show that the intracellular domain of the quantum dots intensity is only about 0.10 of that in the intercellular region.

\section{Conclusion}

Our results show that the smaller fluorescein molecules can be delivered transdermally without the chemical enhancer oleic acid. However, fluorescein transport is further enhanced in the presence of oleic acid. On the contrary, the transdermal delivery of quantum dots $7 \mathrm{~nm}$ in size is only effective with oleic acid treatment. Furthermore, unlike the fluorescein molecules, the hydrophilic quantum dots can only be delivered effectively through intercellular pathways. Our results suggest that non-invasive, chemically enhanced delivery methodology may be used to delivery nanometer sized nanocrystals across biological barriers. This observation may be used to image pathological tissues using non-invasively delivered quantum dots. In addition, our observation suggests that the delivery of hydrophilic nanoparticles for therapeutic purposes should be focused through intercellular pathways.

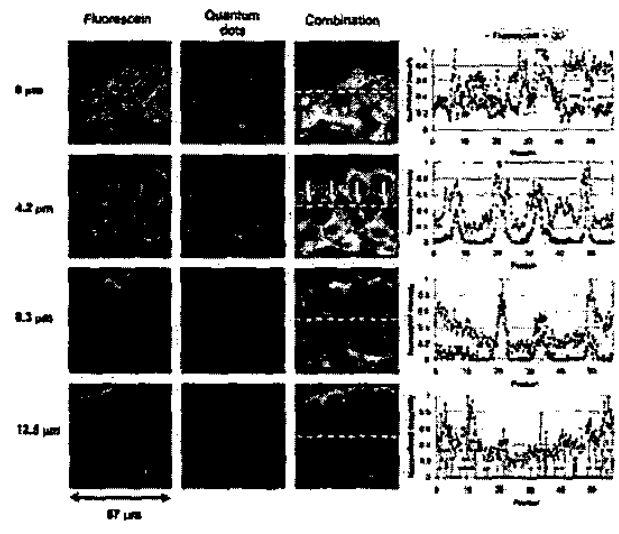

Figure 1 Simultaneous dual color images at different depth. Cross-sectional analyses rightmost panel at the right most reveals the delivery pathway differences between fluorescein and quantum dots.

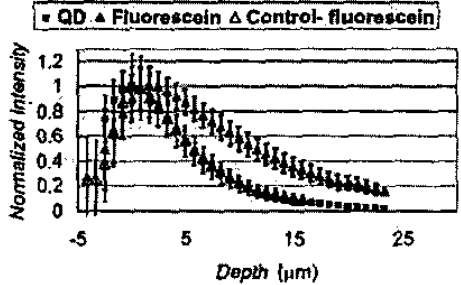

Figure 2 the normalized intensity profile demonstrated even with the facilitation of oleic acid, the $7 \mathrm{~nm}$ OD cannot penetrate skin as deep as the smaller fluorescein molecule do.

\section{References:}

[1] D. R. Larson, W. R. Zipfel, R. M. Williams, S. W. Clark, M. P. Bruchez, F. W. Wise, and W. W. Webb, "Water-soluble quantum dots for multiphoton fluorescence imaging in vivo," Science, vol. 300, pp. 1434-1436, 2003.

[2] B. Yu, K. H. Kim, P. T. C. So, D. Blankschtein, and R. Langer, "Visualization of oleic acid-induced transdermal diffusion pathways using two-photon fluorescence microscopy," Journal of Investigative Dermatology, vol. 120, pp. 448-455, 2003. 